\author{
Submitted: \\ 14.05.2020 \\ Accepted: \\ 18.05.2020 \\ Published: \\ 15.06.2020

\section{Is it safe for patients to apply standardized malignancy risk stratification systems in the management of thyroid} \\ nodules? \\ Zbigniew Adamczewski ${ }^{1,2}$ \\ ${ }^{1}$ Department of Endocrinology and Metabolic Diseases, Medical University of Lodz, Lodz, \\ Poland \\ ${ }^{2}$ Department of Endocrinology and Metabolic Diseases, Polish Mother's Memorial Hospital - \\ Research Institute, Lodz, Poland \\ Correspondence: Department of Endocrinology and Metabolic Diseases, Medical University \\ of Lodz, Polish Mother's Memorial Hospital - Research Institute, Rzgowska 281/289; \\ 93-338 Lodz, Poland; tel./fax: +48 4227111 40; e-mail: zbigniew.adamczewski@umed.lodz.pl \\ DOI: $10.15557 /$ JoU.2020.0026
}

"Everything should be made as simple as possible, but not simpler" Albert Einstein

The most important clinical issue for a practicing endocrinologist is to answer the question whether the presence of a thyroid nodule/nodules or thyroid focal lesion/lesions in the ultrasound scan (US) provides the basis for referring the patient for surgery. During diagnostic procedures, we have to consider whether living with the existing nodule/nodules is a greater threat to the patient than surgical treatment.

The commonly acknowledged diagnostic principles in patients with thyroid focal lesions have been based on US and fine-needle aspiration biopsy (FNAB) for many years. However, therapeutic decisions are usually taken on the basis of FNAB, which, for many physicians, is a reference standard in directing patients for either a surgery or surveillance.

A very dynamic development and increased availability of imaging (not only ultrasound) have led to detection of a large number of impalpable lesions in patients without clinical signs of thyroid disease.

This is also associated with the widespread application of FNAB irrespective of whether patients have low-risk lesions presenting a stable ultrasound pattern or suspicious nodules requiring further management.

This has resulted in the need to implement reasonable FNAB eligibility criteria.
Long-term observations and analyses of cancer risk associated with thyroid lesions of a given US pattern have made it possible to propose various standardized risk stratification systems.

One of the most well-known tools for ultrasound-based classification of thyroid lesions is the Thyroid Imaging Reporting and Data System (TIRADS) ${ }^{(1)}$, published by Horvath et al. in 2009. This US-based classification enables one to distinguish benign lesions from those with suspicious features characteristic of a malignancy.

In the same year, Cibas and Ali published the Bethesda System for Reporting Thyroid Cytopathology (TBSRTC). This system recommends that each report should begin with one of the six diagnostic categories. Each of the categories denotes a different cancer risk that links it to a proper management guideline ${ }^{(2)}$.

These classifications were intended to aid clinical decisionmaking processes, and thereby decrease the number of FNABs and unnecessary thyroidectomies.

In 2017, the European Thyroid Association (ETA) published the novel European Thyroid Imaging and Reporting Data System (EU-TIRADS) based on the guidelines of the ATA (American Thyroid Association), Korean experts and American Association of Clinical Endocrinologists (AACE), and based on the analysis of recent research results ${ }^{(3)}$. 
The simplicity of the EU-TIRADS system is its advantage, and its aim is to facilitate cancer risk stratification in clinical practice. This system enables us to abandon a decision about FNAB in patients with pure cysts and entirely spongiform lesions (EU-TIRADS category 2 - risk of malignancy (RM) close to $0 \%$ ).

In the remaining groups, the key criterion for FNAB is a different size of thyroid focal lesions depending on their US pattern (US-TIRADS category):

- EU-TIRADS 3 (low-risk category): ovoid, smooth isoechoic/hyperechoic, no highly suspicious characteristics; RM: $2 \%-4 \%$, recommendation for biopsy only for nodules $>20 \mathrm{~mm}$.

- EU-TIRADS 4 (intermediate-risk category): ovoid, smooth, mildly hypoechoic, no highly suspicious characteristics; RM: 6\%-17\%, recommendation for biopsy usually for nodules $>15 \mathrm{~mm}$.

- EU-TIRADS 5 (high-risk category): at least 1 of the following highly suspicious characteristics: irregular shape (taller-than-wider shape), irregular margins, microcalcification, marked hypoechogenicity (and solid); RM: $26 \%-87 \%$, recommendation for biopsy for nodules $>10 \mathrm{~mm}$.

The aim of all classifications and scales used in medicine, including EU-TIRADS and TBSRTC for thyroid nodules, is to simplify the communication of results, and thereby increase the interobserver reproducibility of clinical decisions.

The adoption of these principles is dictated by the good of the patient, but it also simplifies the decision-making process. It is, however, this simplification that may be a source of errors.

As for solid lesions (or also with minimal cystic changes), the risk of malignancy evaluated with ultrasound increases when several suspicious US features are identified, but it never reaches $100 \%$. That is why, US can never be the basis for a diagnosis of a thyroid malignancy and must be supplemented with a cytological evaluation. However, it must not be forgotten that in the case of a diagnosis of a malignant lesion, a US image classified to a high-risk category is usually consistent with the US pattern for papillary thyroid carcinoma (PTC). PTC is the most common malignant tumor of the thyroid gland. It is a cancer characterized by well-defined rules of diagnosis, treatment and monitoring (follow-up). One of the most important features of PTC is a very good prognosis because of its slow growth and spread. That is why a great majority of PTC cases are diagnosed in stage T1a, i.e. when its diameter does not exceed $10 \mathrm{~mm}^{(4)}$.

The application of the ETA guidelines for EU-TIRADS 5 lesions will result in the fact that patients with PTC will be deemed eligible for treatment in stage T1b. According to the current guidelines for thyroid carcinoma treatment, these patients must undergo total thyroidectomy, and subsequently frequently adjuvant radioactive iodine $\left({ }^{131} \mathrm{I}\right)$ therapy ${ }^{(5)}$.
On the other hand, treatment of stage pT1a PTC may end after lobectomy, which carries a significantly lower risk of complications $^{(5)}$. Moreover, the effect of the size of the cancer focus on worse course and long-term prognosis should be considered as well ${ }^{(6,7)}$.

The unfamiliarity with these facts combined with thoughtless usage of the EU-TIRADS system will make it impossible to establish a diagnosis at the initial stage of the disease (focus $\leq 10 \mathrm{~mm}$ ), thereby depriving patients of an opportunity for less aggressive treatment and exposing them to potential complications and worse long-term prognosis.

In our country, the Guidelines of the Polish National Societies for the Diagnosis and Treatment of Thyroid Carcinoma recommend biopsy for lesions that are 5-10 $\mathrm{mm}$ in diameter if suspicious features are observed on ultrasound. However, according to these guidelines, there are indications for FNAB of a thyroid lesion $\geq 1 \mathrm{~cm}$ in at least one dimension even if they do not show any increased risk of malignancy ${ }^{(5)}$.

The application of these principles enables PTC T1a diagnosis, but does not reduce the number of biopsies of lesions demonstrating a benign ultrasound pattern. This may suggest that the optimal solution would be to utilize both guidelines when deciding about FNAB.

The simplified use of the EU-TIRADS scale may also lead to the overlooking of false negative results in FNAB. Irrespective of the EU-TIRADS category, each focal lesion for which FNAB is ordered may be diagnosed as benign after cytological evaluation (TBSRTC category 2). This means that some physicians do not take into account a possibility of an error and abandon a decision about surgery despite clinical and ultrasonographic indications.

Altered thyroid structure, visible on US as a focal lesion, especially for foci with a structure that is distinctly different from normal thyroid echogenicity, is associated with a change of the morphology of thyroid follicular cells, the presence of cells that are not observed in the healthy thyroid gland, e.g. lymphocytes, macrophages, granulocytes, and changes in the colloid content.

The presence of constituents only typical of the healthy thyroid gland may mean that a sample has been collected from beyond the pathological focus. In this case, it is necessary to re-analyze the US pattern of the lesion in the context of the full cytological examination. Focusing only on the consecutive steps of the diagnostic algorithm may make us lose the global picture of the disease. This is possible especially when US is used only for assessing eligibility for FNAB.

To sum up, the basis for making a correct diagnostic and therapeutic decision is the complementary application of data obtained from US and FNAB, essentially on the background of the clinical context ${ }^{(8)}$. Only a personalized 
diagnostic approach based on algorithms, rather than their mechanical usage, guarantees optimal therapeutic decisions. However, to make these actions beneficial and, above all, safe to the patient, thorough knowledge regarding principles that underlie the creation of medical scales is needed.

\section{References}

1. Horvath E, Majlis S, Rossi R, Franco C, Niedmann JP, Castro A et al.: An ultrasonogram reporting system for thyroid nodules stratifying cancer risk for clinical management. J Clin Endocrinol Metab 2009; 94: 1748-1751.

2. Cibas ES, Ali SZ: The Bethesda System for reporting thyroid cytopathology. Thyroid 2009; 19: 1159-1165.

3. Russ G, Bonnema SJ, Erdogan MF, Durante C, Ngu R, Leenhardt L: European Thyroid Association guidelines for ultrasound malignancy risk stratification of thyroid nodules in adults: The EU-TIRADS. Eur Thyroid J 2017: 6: 225-237.

4. Dobruch-Sobczak K, Adamczewski Z, Szczepanek-Parulska E, Migda B, Woliński K, Krauze A et al.: Histopathological verification of the diagnostic performance of the EU-TIRADS classification of thyroid nodules-results of a multicenter study performed in a previously iodine-deficient region. J Clin Med 2019; 8: 1781.

\section{Conflict of interest}

The author does not report any financial or personal connections with other persons or organizations, which might negatively affect the contents of this publication and/or claim authorship rights to this publication.
5. Jarząb B, Dedecjus M, Słowińska-Klencka D, Lewiński A, Adamczewski Z, Anielski R et al.: Guidelines of Polish National Societies diagnostics and treatment of thyroid carcinoma. 2018 update. Endokrynol Pol 2018; 69, 34-74.

6. Zhang TT, Li CF, Wen SS, Huang DZ, Sun GH, Zhu YX et al.: Effects of tumor size on prognosis in differentiated thyroid carcinoma smaller than $2 \mathrm{~cm}$. Oncol Lett 2019; 17: 4229-4236.

7. Sun W, Lan X, Zhang H, Dong W, Wang Z, He L et al.: Risk factors for central lymph node metastasis in $\mathrm{CN} 0$ papillary thyroid carcinoma: a systematic review and meta-analysis. PLoS ONE 2015: 10: e0139021.

8. Adamczewski Z, Lewiński A: Proposed algorithm for management of patients with thyroid nodules/focal lesions, based on ultrasound (US) and fine-needle aspiration biopsy (FNAB); Our own experience. Thyroid Res 2013; 6: 6 . 